

\title{
Represa Salvajina: lejos del desarrollo, cerca del olvido. 1985-2016
}

\author{
Por Yurany Alexandra Pérez Lugo* y Lenin Gabriel Díaz Escandón**
}

Resumen: la represa Salvajina, una de las grandes hidroeléctricas de Colombia, ubicada en el noroccidente del Cauca, en los municipios de Suárez, Morales y Buenos Aires, ha sido una construcción controversial por los impactos sociales y ambientales que ha generado durante la administración de diferentes multinacionales y empresas privadas. La gobernanza se involucra en la administración de esta, pues es permanente la articulación entre Estado sociedad civil y actores privados, donde supone el reto de efectuar la gobernabilidad en el margen legal.

La administración de la represa, sus repercusiones socioambientales y la gobernabilidad son tres factores que confluyen para determinar el impacto general de este proyecto frente a la población aledaña. A partir de lo anterior, este escrito intenta ver cómo dicho impacto ha sido remediado, o, en su defecto, cuáles son las tentativas para lograrlo, pues desde una perspectiva críticoanalítica, el manejo y regulación del Estado frente a los actores privados permite que las dinámicas sociales se vean impactadas negativa o positivamente, en la culturalización de nuevas formas de vida relacionadas con la construcción de la represa. En suma, el artículo apunta a que el reto del Estado, en el marco de la gobernanza, y dentro de una armonía teórica horizontal, es suscitar la gobernabilidad entre los actores que buscan beneficio de distinta índole en la represa Salvajina.

* Estudiante de ciencia política Universidad del Cauca. Pertenece al Grupo de Estudios Lingüísticos, Pedagógicos y Socioculturales (GELPS). Correo electrónico: yuliamsalas9@gmail.com

** Estudiante de ciencia política Universidad del Cauca. Pertenece al Grupo de Estudios Linguiísticos, Pedagógicos y Socioculturales (GELPS). 
Palabras clave: megaproyectos, desarrollo, impacto social, impacto ambiental, actores privados, sociedad civil, acción estatal, gobernanza, gobernabilidad.

\section{Salvajina Dam: far from development, close to oblivion}

Abstract: Salvajina dam, one of the greatest hydroelectric of Colombia, located in the Northwest of Cauca, in the municipalities of Suárez, Morales and Buenos Aires has been controversial for the social and environmental impacts generated, within the administration of different multinationals and private companies. Governance is involved in the administration of this, because joint is permanent between State, civil society and private actors, where the challenge of governance in the legal margin.

The management of the dam, its social and environmental impacts; and it governance are three factors that converge in determine an impact general to the population surrounding and how these have been remediated, or in its defect them attempts of achieving it. As a perspective critical analytical, management and regulation of the State to private players allows that the social dynamics will be impacted positively or negative on the culturalisation of new forms of life because of the construction of the dam. Raise governance is the challenge of the State within the framework of governance, within a horizontal theoretical harmony between players seeking benefits of different kind in the Salvajina dam.

Keywords: Megaprojects, development, social impact, environmental impact, private actors, civil society, state action, governance, governability.

Cómo citar este artículo: Pérez Lugo, Yurany Alexandra y Díaz Escandón, Lenin Gabriel (2017). Represa Salvajina: lejos del desarrollo, cerca del olvido. 19852016. Revista Controversia, 208, 283-314.

Fecha de recepción: 1 de febrero del 2017 Fecha de aprobación: 8 de abril del 2017 


\section{Introducción}

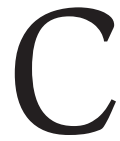

on el fin de proporcionar una mirada amplia de la incidencia de la represa de Salvajina en los municipios de Suárez, Morales y Buenos Aires, desde las contradicciones que el desarrollo genera mediante los impactos sociales y ambientales, se ha recurrido a un estudio de carácter histórico y coyuntural, de tipo descriptivo y analítico, que recopila fuentes secundarias en informes de organizaciones como el Cric y el PCN, además de bibliografía académica de autores como Arturo Escobar, Tatiana Roa Avendaño, Axel Rojas y Érika González.

Actualmente, una de las problemáticas más acuciantes en el territorio colombiano es la frecuente violación a los derechos humanos debido a los ideales del desarrollo económico. Dicho proceso ha generado desplazamiento forzado, y otras formas de violencia, que las empresas aprovechan para su propio beneficio, a costa de acabar no solo con los ríos, sino también con las comunidades que subsisten gracias al afluente hídrico. Carlos Rosero (2002), demuestra que los procesos de desplazamiento van más allá de las dinámicas del conflicto, pues también aluden a procesos de desarrollo económico:

Una cosa sabemos a ciencia cierta y es que la noción imperante de desarrollo y a quienes la instrumentalizan en su beneficio les importa muy poco qué y cómo atropella. El desplazamiento forzado interno [...] no es cosa aislada, sino un conjunto de acciones sistemáticas, abiertas y deliberadas y, por lo tanto, inscritas y funcionales no solo a la dinámica de la guerra, sino también a la concepción de desarrollo (Rosero, citado por Escobar, 2005, p.47).

Formas de opresión estatal como las fuerzas militares y paraestatales como grupos paramilitares, constituyen un instrumento importante al que el Gobierno nacional ha recurrido para ejercer violaciones de derechos humanos (Osorio y Betancur, 2013), pues el interés económico de los gobiernos, está en contra de las prerrogativas del pueblo, quienes, a través 
de la expresión social, que generalmente se acompaña de actos violentos, han encontrado una opción en contra de los megaproyectos económicos.

Los impactos sociales, de los que surgen los pronunciamientos de la sociedad civil, son evidentes, pues tienen implicaciones directas, generalmente a corto plazo, para quienes las experimentan, $\mathrm{n}$ radicalmente sus condiciones de vida. A diferencia de los impactos sociales, los impactos ambientales poseen un carácter discreto y su progreso no es acelerado; pero a largo plazo, solventarlos implica procesos de gran magnitud como reforestación, control de residuos, repotabilización de agua, entre otros, y no hacerlos acarrearía cambios irreversibles en el ambiente y en la forma de vida de la sociedad civil.

Debido a que los conflictos ambientales no son recientes, sino un producto del proceso histórico en el que han intervenido varias dinámicas sociales y diversos actores, cabe mencionar que algunos de ellos son las compañías multinacionales, los grupos armados ilegales y la sociedad civil mediante líderes sociales.

Para el caso de la represa de Salvajina y respecto al carácter históricocoyuntural de dicho proyecto, hay que tener en cuenta que este generó el desplazamiento forzado de alrededor de 10000 personas dedicadas a la agricultura, según los datos del Movimiento Ríos Vivos (2015). Además, originó la obstaculización de caminos que campesinos, indígenas y afrodescendientes habían construido para interconectar el territorio, caminos que hoy, en su mayoría, siguen aislados y con precarias condiciones de servicios públicos (Meneses, 2013). Las situaciones anteriores muestran desde el desarrollo económico como promotor de la construcción de la hidroeléctrica donde:

muchas veces [...] las que menos terminan beneficiadas son las comunidades directamente afectadas por el proceso, en el caso de Salvajina se generaba energía eléctrica para muchas ciudades y la gente que estaba 
alrededor ni siquiera tenía acceso a ese servicio" (Corredor, citado por Meneses, 2013).

Problemáticas como la falta cobertura del servicio de energía en la zona donde es producida, así como distintas variables que impactan el ambiente y la sociedad, como las prácticas productivas o la movilidad, son el centro de la discusión en lo que respecta a la gobernanza y a la gobernabilidad, conceptos que se abordarán teóricamente más adelante.

En suma, el presente artículo se divide en tres secciones en las que se tratarán, en primera instancia, los temas del contexto mundial y local respecto a la construcción de la represa de Salvajina. En segunda instancia, se abordarán los elementos de su administración, los impactos socio-ambientales y las perspectivas de la gobernanza, a partir de las funciones conjuntas de actores privados y el Estado para y con la sociedad civil. Finalmente, se analizará la gobernabilidad dentro de este contexto. Lo anterior, para determinar cómo estos aspectos en su conjunto, son indicadores de las contradicciones propias de la construcción de megaproyectos energéticos en función del desarrollo económico.

\section{Hidroeléctricas y el desarrollo}

Entender el deber ser del proyecto de infraestructura de una hidroeléctrica, los motivos por los cuales se construye y sobre los cuales se aspira impactar la región, en teoría de forma positiva y mitigando los impactos adversos, son necesarios para obtener un punto de partida desde el cual analizar las contradicciones que la construcción de una hidroeléctrica provoca.

Para los años ochenta, las reformas estructurales del Consenso de Washington se adherían a las agendas de Gobierno de ese entonces y buscaban el desarrollo y la estabilidad política y económica en América Latina; sin embargo, gracias a que las reformas no tenían en cuenta las condiciones de cada país, se generaron profundas transformaciones que transitaron de "regímenes autoritarios y regulados a regímenes desregu- 
lados, democráticos y orientados por el sistema del mercado" (Lechini, 2008,p.11).

La expansión capitalista, generada por el desarrollo, demandaba la extracción de materias primas, las cuales requerían exorbitantes cantidades de energía, obtenida, principalmente, de las hidroeléctricas, pues las agotadas reservas de hidrocarburos y los altos precios del combustible a causa de la crisis del petróleo, demandaban formas efectivas y económicas de producir energía. (Roa Avendaño y Duarte, 2013).

En los años sesenta y setenta, la energía hidroeléctrica comprendía un campo innovador en el ámbito mundial, dicho campo se concentró en América Latina, pues su situación geográfica le favorecía. Esto llamó la atención de países desarrollados como Estados Unidos, porque vieron condiciones favorables en el territorio latinoamericano para la construcción de represas. Brasil se consolidó como el mayor productor de energía hidroeléctrica en los años ochenta. Este país concentraba el 91 \% de la energía producida; en menor escala, se construían represas en Argentina, Colombia, México, Chile, Perú y Venezuela (Schorr, 1984).

El discurso del desarrollo se trasladó a la institucionalidad en el año de 1997, cuando el Banco Mundial junto a la Unión Mundial Para la Naturaleza, los actores gubernamentales y privados, las instituciones financieras, la sociedad civil y los afectados por las represas de distintos países consolidaron la Comisión Mundial de Represas, con el fin de:

revisar la eficacia de las grandes represas para promover el desarrollo y evaluar alternativas para el aprovechamiento del agua y la energía. Formular criterios aceptables internacionalmente, donde fuera adecuado guías y normas, para la planificación, diseño, evaluación, construcción, funcionamiento, inspección y desmantelamiento de represas (CMR, 2000, p.2). 
La CMR promovía las represas como un medio importante "para satisfacer necesidades sentidas de servicios de agua y de energía y como inversiones estratégicas a largo plazo con capacidad para proporcionar múltiples beneficios" (CMR, 2000, p.56). Desde esta perspectiva, un proyecto de represa ostentaba el término de unidad y sostenibilidad regional, como fuente de ganancias y de desarrollo humano; su construcción reducía brechas de desigualdad por medio del desarrollo de la agricultura, el aumento en el cubrimiento de agua y energía, la disminución de inundaciones, el desarrollo de la alimentación rural, el desarrollo acuícola y, en los países secos, la mejora del clima tropical (CMR, 2000).

Es pertinente aclarar que la CMR promulgó estos impactos como positivos, a propósito de casos de países desarrollados como Canadá, Noruega o Rusia; al mismo tiempo, impulsó la construcción de hidroeléctricas en América Latina y África, sin tener en cuenta el factor diferencial de los contextos sociales y económicos de los países en mención. De esta manera, la CMR centró la discusión en las "capacidades" que los países subdesarrollados poseían para generar energía a partir de las hidroeléctricas; no obstante, se apoyó en datos obtenidos de países desarrollados como EE. UU, principalmente.

La analogía entre los intereses presentados por actores políticos y privados impulsados por el desarrollo capitalista y el discurso desarrollista estipulado en la CMR permitirán evidenciar que, a pesar de que las represas traían beneficios, los impactos negativos se presentarían en mayor magnitud, y, de no ser contrarrestados, modificarían drásticamente el entorno donde se instalara una represa, de tal forma como lo expresa Escobar, "tanto la modernidad como el desarrollo son proyectos espaciales y culturales que exigen la conquista incesante de territorios y pueblos, así como la transformación ecológica y cultural” (Escobar, 2005,p.48). 


\section{La Salvajina, luchas sociales y administración, 1983-2004}

El departamento del Cauca ha contado con presencia de diversas empresas multinacionales. Debido a su rica biodiversidad, este departamento es atractivo para la explotación de recursos naturales. Según datos consultados, hasta el año 2005, existían quince hidroeléctricas, la mayoría ubicadas en la cuenca del río Cauca, ejecutadas o a punto de serlo, como se evidencia en la siguiente tabla.

\section{Tabla 1. Hidroeléctricas en el Cauca hasta el 2005}

\begin{tabular}{l|c|c}
\multicolumn{1}{c|}{ Nombre } & Tipo & Estado \\
\hline Brazo Seco & Pequeña central hidroeléctrica & Proyectada \\
\hline Descanse & Microcentral hidroeléctrica & Proyectada \\
\hline Buco & Microcentral hidroeléctrica & Proyectada \\
\hline Jolí & Central hidroeléctrica & En operación \\
\hline Asnazú & Central hidroeléctrica & En operación \\
\hline Río Palo & Central hidroeléctrica & En operación \\
\hline Salvajina & Central hidroeléctrica & En operación \\
\hline Sinaí & Central hidroeléctrica & En operación \\
\hline Sajandí & Central hidroeléctrica & En operación \\
\hline Coconuco & Central hidroeléctrica & En operación \\
\hline Patico & Central hidroeléctrica & En operación \\
\hline Florida & Central hidroeléctrica & En operación \\
\hline Ovejas & Central hidroeléctrica & En operación \\
\hline Andalucía & Central hidroeléctrica & Proyectada \\
\hline
\end{tabular}

Fuente: Centro de Estudios Interculturales de la Pontificia Universidad Javeriana de Cali. Recuperado de http://www.javerianacali.edu.co/sites/ujc/files/node/ fielddocuments/field_document_file/analisis_posesion_territorial__tensiones_ interetnicas_e_interculturales_en_el_cauca_1.pdf 
La invasión territorial por parte de foráneos a estas zonas rurales, con población mayoritariamente afro (Suarez y Buenos Aires) y predominancia indígena (Morales), para edificar hidroeléctricas, implica hablar de la naturaleza y de lo sagrado. La invasión-despojo de la naturaleza, constituida por la tierra y el agua en este contexto, involucra asumir nuevas dinámicas por parte de las formas vivientes existentes, que provocan, además de resistencias, transformaciones socioculturales y migraciones (Zapata, et al, 2013).

Al presentarse una fuerte influencia de actores privados o empresas multinacionales que llegan a estos territorios, se crea un escenario conflictivo, pues su intención de una nueva organización política y económica, en función del desarrollo o crecimiento económico de la región, desconoce el espacio natural desde los sentidos amplios, como la integralidad, la cosmovisión y el significado, que se han construido por parte de los habitantes locales (Galvis, 2014).

El papel del Estado como mediador en este tipo de escenarios conflictivos asume una paradoja, ya que se debate entre el componente institucional, es decir, sus fines con la sociedad, y el componente neoliberal de las dinámicas que el mercado global exige. Respecto al primer componente, según la Constitución Política, se establece que los fines del Estado son:

servir a la comunidad, promover la prosperidad general y garantizar la efectividad de los principios, derechos y deberes consagrados en la Constitución; facilitar la participación de todos en las decisiones que los afectan y en la vida económica, política, administrativa y cultural de la Nación; defender la independencia nacional, mantener la integridad territorial y asegurar la convivencia pacífica y la vigencia de un orden justo (Constitución política de Colombia, 1991).

Si se revisan los fines anteriormente descritos, parece que el Estado no es congruente con ellos, ya que, más que estar al servicio de los co- 
lombianos, está al servicio de la economía global, cuando se trata de la construcción de megaproyectos económicos. Dichos proyectos representan un claro riesgo de pérdida de tierras y de degradación del ambiente; sin embargo, el Estado muestra una preferencia por dejar lo público al servicio de lo privado, de los grandes capitalistas y las multinacionales, para quienes impera un modelo de desarrollo que solo los beneficia a ellos y, por ende, degrada y limita a la comunidad. Lo anterior es asociable a las dinámicas propias del Delito Económico Organizado DEO, por medio del cual, una organización económico-delictiva acciona desde lo legal-ilegal, pues existe un lazo social entre instituciones del Estado y actores privados que lo permite y lo alienta, estos actores, motivados por la adquisición de capital, crean y reproducen relaciones de dominación y control social que, lejos de castigar estos comportamientos, los ponen en el camino de la impunidad (Pegoraro, 2013).

\section{Gráfica 1. Ubicación geográfica de los municipios de Suárez, Morales y Buenos Aires}

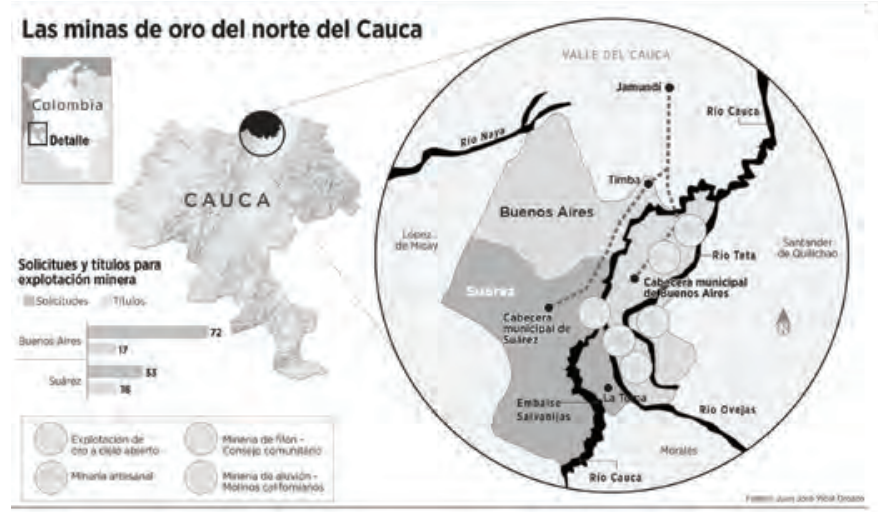

Fuente: El Espectador. Recuperado de http://static.elespectador.com/archivos/2012/03 /0db719fa0f627e2c52ebc39ee6a97a26.jpg

La alianza entre el poder político y el poder territorial está íntimamente ligada al poder económico que trae consigo el desarrollo, porque este 
obstruye la verdadera intervención social hacia las comunidades locales. Por eso, la deficiente actuación del Estado termina por satisfacer los intereses de los entes dominantes, esta es la razón de que ni la acción pública ni la del Gobierno sean neutras en la toma de decisiones (Roa Avendaño y Duarte, 2013).

Ahora bien, es tiempo de observar las especificidades geográficas de la represa de Salvajina, para entender el centro del conflicto que la rodea. La hidroeléctrica está ubicada en el municipio de Suárez, zona montañosa entre las cordilleras Central y Occidental, allí predominan la economía campesina y la minera. Por su parte, Suárez limita con Buenos Aires, Morales y López de Micay (ver imagen 1). Tanto Buenos Aires, como Suárez y Morales son territorios eminentemente campesinos de poblaciones afrodescendientes, nativos indígenas y mestizos que cuentan con el rio Cauca y el rio Ovejas como los principales afluentes hídricos (CRC, 2010).

Durante el siglo Xx y en lo corrido del XXI, según Guzmán y Rodríguez (2015), la región norte caucana ha sido impactada notablemente por los siguientes procesos socioeconómicos: 1) una fuerte expansión de la industria del azúcar y del etanol, 2) el desarrollo de una industria de punta, apoyada por el Estado con la Ley Páez, 3) la construcción de la hidroeléctrica Salvajina, 4) una importante economía ilegal y, finalmente, 5) el resurgimiento de la minería y el sostenimiento de economías campesinas (p.43).

Desde mediados de los años cincuenta, el embalse de Salvajina ya contaba con estudios que daban vía libre a su construcción; sin embargo, fue en 1985 cuando se decidió edificar el embalse por parte de la Corporación Autónoma Regional del Valle del Cauca (cvc) con recursos provenientes del Estado colombiano y otra parte con los recursos del 
municipio de Buenos Aires. Hoy la Salvajina es jurisdicción del municipio de Suárez ${ }^{1}$.

Dos años antes de la construcción de la represa, se expusieron los propósitos que justificaban la ejecución del proyecto. Estos argumentos giraban en torno a la mitigación de las constantes inundaciones generadas en la zona, así como también a la producción de energía hidroeléctrica (Guzmán y Rodríguez 2015, P.42). Las comunidades asentadas en los municipios beneficiarios de este proyecto obtendrían inversión social para la construcción de obras públicas en el casco urbano de Suárez, dotación de medios de transporte terrestre y fluvial, crédito para producción agrícola, provisión de empleo, electrificación, compra de tierra para la reubicación de quienes vivían en zonas que serían utilizadas, puestos de salud, construcción de puentes, acueductos veredales y escuelas (Escué, 1995).

Las movilizaciones por parte de los pobladores aledaños a la represa datan desde el año 1986, producto del incumplimiento de las múltiples promesas, además por la advertencia que Asoprodasa² entregó sobre las posibles y nefastas consecuencias ambientales y sociales que generaría este proyecto. La primera movilización fue un hito en las presiones a los estamentos gubernamentales: la marcha dirigida hacia Popayán, Cauca, estaba conformada por 5000 personas que exigían el despliegue de una negociación, lo que dio como fruto el primer compromiso firmado en 1986. Este, adicional al firmado en 1983, comprometía a los

1 Este cambio geopolítico ocurrió para diciembre de 1989. Cuando Suárez aún era corregimiento de Buenos Aires, líderes comunitarios crearon una junta bajo la denominación de Pro Desarrollo con el propósito de impulsar la creación de Suárez como otro municipio del departamento del Cauca, el cual presentaba una tendencia de prosperidad como producto del auge aurífero, inundado ulteriormente por la Salvajina. La propuesta fue aprobada bajo la Ordenanza 013 del año en mención y la represa pasó a ser jurisdicción de Suárez (Juanillo, 1997)

2 Asociación Pro Damnificados de la Salvajina Asoprodasa, conformada por quienes se identificaban como indígenas, afros y campesinos, estaban estudiando las repercusiones de la represa desde 1983 (Escué, 1995). 
responsables a mitigar los impactos del proyecto (Ararat, et al., 2013, citado en Caicedo, 2017).

Cabe mencionar que para la construcción del embalse no solo se usaron las aguas del río Cauca, sino también las de los ríos Inguitó, Asnazú y Marilópez, Damián, Marilopito, y las de las quebradas El Chupadero, La Chorrera, Los Pasos, Los Morados, La Laja, El Danubio y San Miguel, por último, se usaron también las aguas del río Ovejas, con la finalidad de repotenciar la generación de energía, lo cual implicaba desviar el $90 \%$ de su caudal promedio. Para llevar a cabo este propósito, los informes presentados justificaban la desviación, con la premisa de que este río era el causante de las constantes inundaciones en la zona (Corporación servicios comunitarios sembrar, 2010).

Entre tanto, la historia de la administración del embalse demuestra que este ha pasado por manos de diversas empresas tanto nacionales como extranjeras. Hasta 1995 la represa fue administrada por la CVC, para luego pasar a manos de la empresa colombiana de Energía del Pacífico -Epsa-, la cual fue creada con el propósito de responder a la independización de las funciones ambientales y comerciales correspondiente al negocio eléctrico ejecutado en un inicio por la cvc. Posteriormente, como lo indica la agencia de comunicación perteneciente al periódico virtual Colombia Informa (Colectivo Punto de Fuga, 2012), el 5 de junio de 1997 el Gobierno nacional vendió en subasta pública el 56,7\% de la propiedad accionaria de Epsa a un consorcio formado por Houston Industries y Electricidad de Caracas. En el año 2000, este consorcio cedió su participación accionaría a la multinacional española Unión Fenosa.

En el año 2004, la multinacional española Unión Fenosa revivió la propuesta de la desviación del caudal del rio Ovejas, rechazada por la comunidad en la consulta previa efectuada en 1997. El Ministerio de Ambiente a la cabeza de Sandra Suárez Pérez, durante la presidencia de Álvaro Uribe Vélez, omitió los estudios realizados años atrás sobre el perjuicio social y económico que traería el desvío del río y, finalmente, 
otorgó la autorización a la petición, lo cual ocasionó una creciente violación a los derechos a las comunidades en toda su magnitud: violación al derecho de respeto de la cultura étnica, a la ancestralidad del territorio, al habitad, a la consulta previa y a la vida.

Para marzo del año 2009, las acciones adquiridas por la multinacional Unión Fenosa pasaron a otra corporación española llamada Gas Natural Fenosa, la cual tiene una gran cobertura mundial. Hasta diciembre del mismo año, la hidroeléctrica estaba bajo la dirección de Epsa y Gas Natural Fenosa; sin embargo, durante el mismo periodo el $100 \%$ de las acciones se adjudicaron a Colener S.A.S. sociedad perteneciente a Colinversiones S.A. (Colectivo Punto de Fuga, 2012)

\section{Contexto socioambiental en los municipios de Suárez, Morales y Buenos Aires}

El siguiente apartado del documento busca caracterizar los aspectos más relevantes del contexto social y ambiental de los municipios de Suárez, Morales y Buenos Aires, Cauca, en torno al impacto generado por la represa de Salvajina. Según proyecciones del Dane, estos tres municipios representan el 5,6\% de la población del departamento del Cauca, es decir, 77611 habitantes de un total de 1391889 que, por su ubicación territorial, se encuentran próximos a la represa de Salvajina.

Para los años ochenta, previo a la construcción de la represa, se requería el desalojo de los terrenos donde se llevaría a cabo esta obra. Eran, a saber, 2124 hectáreas habitadas por indígenas y afrocolombianos en su mayoría. La totalidad de las comunidades que allí se encontraban se vieron obligadas a desplazarse a territorios aledaños, zonas altas de la montaña o a la ciudad de Cali —destino de la mayoría- (Sulé, 2006). Dicha migración se dio en condiciones de pobreza y favoreció el desempleo y la falta de condiciones dignas de vida. 
El traslado a zonas de alta montaña significaba para los habitantes transformar sus modos de producción junto con los cultivos que habitualmente plantaban, pues, como señala el Censat Agua Viva (2009), los predios posteriormente ocupados por el embalse de Salvajina funcionaban como sustento económico para indígenas y afrocolombianos nativos, porque en estos se desarrollaban actividades como pesca, agricultura, balsería y minería. Lo anterior dio paso a una crisis por parte de las fincas asentadas en estos territorios, pues debían ser inundadas, y esto limitaría de manera abrupta el sustento económico y la posibilidad de generar nuevas actividades o actividades similares a las anteriores, aunque en distintas condiciones.

Eduard Mina, líder comunitario perteneciente al Proceso de Comunidades Negras PCN, oriundo de la Toma-Cauca y afectado por la construcción de la represa ya mencionada, denuncia que, más allá del desplazamiento que sufrió la sociedad civil, fueron evidentes las rupturas entre las etnias nativas internamente y en conjunto entre indígenas y afros, pues varias familias quedaron separadas por el embalse, lo cual hacía imposible movilizarse entre poblaciones a las que anteriormente se llegaba de manera sencilla. Este es el caso de los corregimientos Mindalá y Pureto, donde se evidenció la ruptura en la identidad cultural de la región (Colectivo Punto de Fuga, 2012).

Esta ruptura étnica se dió porque previamente los habitantes habían construido un "arraigo por el territorio", noción que Escobar (2000) califica como una entidad multidimensional, donde diferentes prácticas y relaciones están determinadas por el espacio y su sistema simbólico/ cultural que, para el caso que nos atañe, se fraccionó a partir de la construcción de la Salvajina. Este hecho u otro que violente el territorio reconfiguran los simbolismos culturales étnicos.

Las prácticas de producción locales parten también de este conjunto de relaciones. Respecto a la minería productiva Eduard Mina refiere: 
Las mejores minas de oro del municipio de Suárez están sumergidas en Salvajina. La parte más productiva de Suárez, que eran las laderas del río Cauca, igual quedaron navegados $32 \mathrm{~km}$, lo que ha ocasionado una gran afectación en la parte agrícola en el municipio (Colectivo Punto de Fuga, 2012).

La minería en el río posee particularidades económicas y culturales. Económicas, en tanto generan mercado de consumo local, generalmente sostenible, y se ven acompañas de prácticas agrícolas; culturales, en tanto el río y la práctica minera son simbólicas, pues constituyen un sistema tradicional de producción en las comunidades ribereñas (Escobar, 2010). En ese sentido, el señalamiento de Eduard Mina evidencia la transformación económico-cultural de los pobladores, quienes asumían una forma de vida en torno al río Cauca.

Las nuevas prácticas agrícolas en zonas de alta montaña, que remplazarían a la minería o a la agricultura en las orillas del río Cauca, son parte de nuevos imaginarios económicos, que responden en forma de resistencia a las prácticas de globalización neoliberal, mediante una dimensión económica alternativa basada en el lugar y sus prácticas condicionadas a la lógica de mercado global, gracias a la concepción de mercado local a las que se acogen estas prácticas productivas (Escobar, 2005).

Entre tanto, en las veredas aledañas al municipio de Suárez y Morales, los habitantes transportaban sus productos hacia los planchones operados por Epsa, los cuales partían de dos únicos lugares: las veredas Santa Bárbara y San José; sin embargo, el acceso a estos puestos de cargue se vieron afectados, pues la accesibilidad se dificultaba por el estado de la vía, aspecto que Epsa tenía que garantizar. Había múltiples derrumbes, nula intervención de la vía, un único horario de operación de los planchones, temporadas de estiaje y mínima cobertura vial, lo cual retenía la producción y la comercialización de las veredas (Ministerio de Ambiente, 2008). 
El carácter periférico de muchas veredas, casi inaccesibles por la malla vial tanto terrestre como fluvial, no solo afecta el sustento económico de sus pobladores, también afecta su salud y los aspectos más diversos de su vida, pues requieren de esta conexión para desplazarse a las cabeceras municipales de Suárez, Morales y Buenos Aires a recibir los servicios de Salud y Educación (Min Ambiente, 2008). En suma, a esta población se le estaría vulnerando el derecho a la salud y a la educación, lo cual provoca el aumento de las desigualdades sociales a causa del delito económicamente organizado, con los cuales se eximen las responsabilidades que otros procesos económicos legales o no, generan en la sociedad (Bauman, 2000).

El impacto ambiental ocasionado por la construcción de la represa de Salvajina se ha visto opacado por el impacto social que ha ocasionado, ya que este último es más evidente para los pobladores y estudiosos del tema; sin embargo, se habla del impacto al territorio y a la tierra fértil, pues la productividad ha variado a causa del microclima inestable, que ha erosionado el territorio aledaño a la represa. Por su parte, la disminución de las inundaciones por el control del caudal del río Cauca fue evidente solo los primeros años (González, 2008). Entre otras consecuencias a causa de la masiva intervención de los recursos naturales al momento de construir la Salvajina, se reconoce:

La obstrucción del flujo natural de los ríos, la alteración de sus caudales y la inundación de vastas zonas de selvas ha generado la alteración de ciclos de vida y de reproducción animal y vegetal [...] establecimiento de amplias zonas de amortiguación ambiental conducen a la imposición de nuevas vocaciones productivas y usos del suelo (Avendaño y Navas, 2014, p. 116-117).

Eduard Mina también centra su pronunciamiento en el impacto al ambiente, declara que la madera de la zona no llegó a ser removida en su totalidad, además, al momento de abrir la represa los residuos caen con el caudal. Respecto a las inundaciones y el desvío del río Ovejas, Mina señala que el control del caudal se utiliza como excusa para su desvió, 
pues el interés real de Epsa es aumentar la producción de energía. Esta afirmación tiene asidero en la realidad de quienes viven próximos al caudal que despide la represa, pues, a pesar de la desviación del río Ovejas, siguen presentando inundaciones. Mina sostiene que "todo eso se hizo con un fin de produccir y no de controlar" (Colectivo Punto de Fuga, 2012).

No es posible desconocer que Suárez ha sido un territorio con alta incidencia del conflicto armado. La construcción de la represa vislumbraba un gran desarrollo económico, por lo cual la presencia del Ejército fue necesaria, para evitar las acciones armadas por parte de grupos ilegales, quienes incrementaron la violación de los derechos humanos de la población y la criminalización de varios líderes comunitarios - como más adelante se detalla- (González, 2008). El sur de Colombia ha sido un punto estratégico de control territorial por parte de las guerrillas, pues es un área de influencia política vinculada a la historia agraria y campesina de la región, así como al comercio y a la producción de coca. Por su presencia activa, las guerrillas imponen en este territorio un modelo de orden social (Trejos, 2013).

El momento más crítico por la disputa de Suárez fue hacia el año 2000, cuando las guerrillas de las FARC y el ELN atacaron la estación de Policía del municipio, ubicada en el centro de la población, ningún uniformado de la Policía ni guerrillero salió abatido, sin embargo, si hubo grandes daños en la infraestructura y una persona murió. La guerrilla quedó al mando del municipio, pues las fuerzas del Estado se vieron obligadas a retroceder, por consiguiente, la represa pasó a ser controlada por las guerrillas de las FARC y el ELN (Canizales, 2013).

Durante nueve meses estos grupos armados hicieron presencia en el área, pero las Auto Defensas Unidas de Colombia (AUC), al mando de Hebert Veloza, alias HH, también querían tener dominio en la zona, por lo cual entraron a disputarse este territorio. El grupo paramilitar dispuso su campamento en los alrededores de Buenos Aires y concen- 
traron su ofensiva en los corredores naturales de la vertiente baja del río Cauca. Las AUC no eran más que un refuerzo necesario para el Ejército en su objetivo por retomar el municipio de Suárez. Entre tanto, el Ejército ubicó su campamento en el muro de contención de la represa y, en diferentes ataques conjuntos Ejército-AUC lograron replegar a las guerrillas en las altas montañas de Suárez, particularmente en Betulia y Timba (Canizales, 2013).

Por su parte, la multinacional Unión Fenosa ha sido acusada de haber hecho señalamientos calumniosos a los líderes que se oponían al megaproyecto del embalse de Salvajina y usaron la presencia de grupos armados para presionar a la comunidad, como lo indica Osorio (2009):

Unión Fenosa, desconociendo la decisión de la comunidad, comenzó en 2004 una agresiva campaña visitando las 17 comunidades impactadas, para convencerlas de las "bondades del proyecto". Usó como táctica el desprestigio y señalamiento de los líderes comunitarios con falsas acusaciones, en una región con presencia de actores armados.

En datos reportados desde el año 2004 hasta el año 2009 se registraron por lo menos treinta y ocho asesinatos a miembros de la comunidad, aún no se tiene conocimiento de sus coautores, sin contar con las ejecuciones extrajudiciales y las amenazas. Uno de los líderes amenazado y asesinado por su resistencia social fue Benicio Flor Belalcázar. Este hombre luchó ardientemente por la protección de las cuencas de agua dulce en Suárez, Cauca, trabajó en iniciativas de agricultura de la comunidad y participó en la comisión de la verdad para la investigación sobre las masacres y la violación de los derechos humanos en el Cauca, como la masacre de Nilo.

Eider Flor, hijo de Benicio Flor Belalcázar y dirigente indígena, fue amenazado en el año 2015 por las reclamaciones consignadas en la Sentencia T-462A de 2014 a la empresa Epsa y a las autoridades ambientales. Alexa Leonor Mina, Mery Yein Mina y Francia Márquez, integrantes de 
la Movilización de Mujeres por el Cuidado de la Vida y el Territorio Ancestral y del PCN fueron amenazadas en el 2016 por bandas criminales. Cabe resaltar que Francia Márquez es una importante líder social afrocolombiana del corregimiento la Toma, ganadora del Premio Nacional de la Defensa de los Derechos Humanos en Colombia en el año 2015, su comunidad mantiene una lucha constante por la resistencia contra las fuerzas políticas, económicas y armadas que buscan controlar el río Ovejas (Ruíz, Pablo 2012).

Las amenazas y el asesinato a líderes sociales son mecanismos de presión, condicionamiento o coacción utilizados por empresas multinacionales para imponer su voluntad. El progreso y el bienestar se constituyen, en principio, como promesas para la comunidad dentro de la estrategia de despojo de capital que, en el marco del conflicto armado, están relacionadas con acciones subversivas. Esto pone en evidencia que las empresas tienen una postura ambigua pues, de un lado legitiman el discurso del desarrolloy de otro, ejercen presión armada para ingresar a estos territorios de explotación (Osorio y Betancur, 2013).

A modo de conclusión, la construcción de la represa de Salvajina trajo consigo diferentes impactos que van desde los cambios en las prácticas productivas y la movilidad, hasta las experiencias violentas de desarraigo, desplazamiento y muerte. Estas dinámicas son culpables de transmutar el modo de vida en las comunidades aledañas que, a pesar de todo, persiguen la continuidad de un proceso histórico siempre ligado a la concepción de la tierra como centro de la identidad étnica, unida de forma simbólica y material a los procesos económicos y políticos de los movimientos sociales originados en este territorio (Bello, 2004). De este modo la tierra no es una abstracción, sino un referente que busca formas de autorepresentación como un espacio vivido en prácticas y relaciones que lo apropian (Velázquez, citada en Bello, 2004). 


\section{Gobernabilidad en la zona de influencia de la represa la Salvajina}

A continuación, se expondrán las principales medidas que ha tomado el Estado, desde la perspectiva de la gobernabilidad, para contrarrestar los impactos generados por la represa de Salvajina, con el fin de determinar las garantías institucionales que las poblaciones afectadas han tenido para enfrentar dicha situación. Para ello el concepto de gobernabilidad se entenderá como:

un supuesto básico de la idea de que unas instituciones estatales bastante fuertes conducen a la canalización de las demandas de la sociedad civil [...] se da cuando existe una respuesta gubernamental eficiente frente a los problemas de una sociedad [...] la mejoría de la gobernabilidad se lograría por medio de una modernización de las prácticas de gobierno democrático (Launay y González, 2010, p.208).

Cabe agregar que la gobernabilidad es una cualidad del Gobierno que, de acuerdo con el contexto en que se encuentre, asumirá un rol diferente que aporte al fortalecimiento institucional y al buen gobierno, en términos de eficacia y efectividad. Las medidas del Estado parten desde el principio de la precaución, la cual lo obliga a tomar acciones cuando alguna intervención cause impactos de tipo ambiental, económico, jurídico a bienes públicos, a la cultura, la salud o la equidad, en especial, cuando exista un riesgo plausible para la región afectada dentro de la incertidumbre acerca de la magnitud o la probabilidad (Cepal y Patrimonio Natural, 2013).

La Resolución 1248 del 11 de julio de 2008, expedida por el Ministerio de Ambiente, y el Acta de 1986 de la construcción de la represa son los principales pronunciamientos del Gobierno y los actores privados respecto a los impactos socioambientales ocasionados por el embalse. Su pertinencia radica en que se le considera como una respuesta guberna- 
mental frente a un problema social, en función de superarlo mediante su intervención.

Producto de los reclamos de la comunidad el Gobierno firmó la conocida Acta de 1986, en la que prometió escuelas, puestos de salud, carreteras, puentes, y trasporte. Hoy, 26 años después las comunidades continúan afrontando las consecuencias de un negocio hidroeléctrico que ha llenado las arcas de las multinacionales, Houston Industries, Unión Fenosa, Gas Natural SDG S.A y Colinversiones (OMAL, 2012).

En 1986 se expidió el Decreto 3000 para una Comisión de Supervisión y Evaluación del Plan de Desarrollo Integral de la Región de Salvajina, cuya misión consistía en la identificación de las complicaciones suscitadas por la represa a los pobladores de los municipios afectados, además preveía el seguimiento al Plan de Desarrollo Integral de la región.

La comunidad sostiene que no se ha cumplido ni un $80 \%$ de lo pactado en el Acta del 86 y que, además, en tanto manifestaron su preocupación en vías públicas, fueron evidentes la persecución a líderes sociales y las amenazas a representantes, así como la represión por parte del Estado a la comunidad en general. Óscar Elías López, líder comunitario y asesor jurídico del Consejo Regional Indígena del Cauca -Cric-, fue víctima mortal producto de su actividad para impulsar las movilizaciones contra Epsa (González, 2008).

En su defensa, y estancanda toda acción que mitigara los impactos, Epsa argumentó: Los efectos negativos de la represa de Salvajina, contemplados en el Acta, no entraron en el contrato de compra de Epsa. Mientras tanto, sí se benefició de su producción eléctrica y de los ingresos que le proporcionó (González, 2008, p.17).

La responsabilidad legal que Epsa tenía para con los territorios se debía cumplir, a pesar de querer estancar los acuerdos primarios. Para ello la Fundación Epsa inviertó dineros en los municipios de Suárez, Morales 
y Buenos Aires para favorecer el desarrollo local mediante proyectos productivos como compensación a la población afectada. La unión Fenosa fue reconocida internacionalmente en 2007 por sus labores de compensación con el premio European Marketplace de Responsabilidad Social Corporativa. A pesar de ello, los planes presentados por la multinacional estaban en una perspectiva ajena al contexto, en tanto la comunidad afirma que "los planes presentados por ellas para vivir con dignidad, de acuerdo con el respeto al territorio y a su identidad cultural, son continuamente rechazados" (González, 2008, p.17).

Para el año 2008, se expidió la Resolución 1248 del 11 de julio del Ministerio de Ambiente, por la que se presentaron cargos de carácter administrativo contra Epsa. Se argumentaron distintos impactos socioambientales expuestos en el apartado y distintas responsabilidades administrativas frente a las cuales Epsa no ha cumplido o solventado. Para el año 2004, Epsa tenía que haber realizado las siguientes acciones:

1. Construcción y ejecución de un Plan de Manejo Ambiental para la operación de la central.

2. Presentar los criterios bajo los cuales rigen las reglas de operación de todas las estructuras de fondo, rebosadero, niveles máximos y mínimos de operación del embalse y niveles del río aguas abajo del sitio de descarga de casa de máquinas.

3. Presentar un informe consolidado sobre la operación de la Central, en los últimos diez (10) años, donde se presente caudal de entrada, caudal de salida, por las diversas estructuras, niveles del embalse, caudales finalmente descargados aguas abajo del embalse y sus niveles asociados.

4. Presentar los lineamientos técnicos y ambientales, bajo los cuales se realizan las descargas de fondo autorizadas por la CVC. Para los programas de gestión social, se deben tener en cuenta los resultados de la evaluación de los proyectos que ha adelantado la Fundación 
Epsa, con el propósito de garantizar la eficacia y sostenibilidad de estos (Ministerio de Ambiente, 2008, p.4-5).

La sanción buscaba el acatamiento de los anteriores términos, así como aplicar cargos legales por su incumplimiento para con la comunidad por más de cuatro años. Los cargos recibidos por Epsa solo se evidenciaron en esta Resolución, pues no se encuentra material alguno que compruebe su ejecución o desacato, lo cual sesgó el papel que Epsa desempeñó frente a la sociedad de este territorio o, en un caso apartado, el escenario en el que tentativamente jugó Epsa con el Estado para no cumplir con los acuerdos.

Los juegos políticos se hicieron evidentes en situaciones de vulneración de los derechos de los pueblos afro, debido a que se hallaron relaciones estrechas entre las empresas culpables de los perjuicios y las corporaciones que, en teoría, deberían intervenir para la protección de los derechos de las comunidades. En lo consignado en el Periódico El Liberal (Betancourt, 1994), la CVC a cambio de otorgar la licitación para la desviación del río Ovejas, obtuvo recaudos financieros de los que no se brindó ninguna explicación, por ello, el representante de la Cámara para ese entonces Juan José Chaux citó al director de Planeación Nacional, Armando Montenegro para que respondiese sobre el supuesto beneficio real brindado por la CVC al departamento del Cauca.

La empresa Epsa es una empresa privada que no solo está acusada por incumplimientos de las promesas a los habitantes de Salvajina, sino que también tiene una tutela del año 2009 por el Juzgado Primero Administrativo de Buenaventura, en la que se consignan las graves afectaciones ocasionadas en el año 1999 a la comunidad a causa de la represa del bajo Anchicayá, ubicada en el Valle de Cauca. Según los datos suministrados, la hidroeléctrica ocasionó un incalculable perjuicio ambiental que afectó notablemente a las comunidades que habitaban en la zona, es decir, a cerca de 6000 personas que aún no han sido indemnizadas por la empresa. 
La CVC la autoridad ambiental en el departamento, y la entidad que debería intervenir para que la Epsa cumpliera con sus compromisos, se declaró impedida para investigar el problema ecológico sin dar explicación alguna. No obstante, tras indagar el caso se encontró que esta corporación poseía el $16 \%$ de las acciones de la empresa eléctrica siendo la multinacional Unión Fenosa su principal accionaria (Marín Correa, 2011).

La ineficiencia de los mecanismos de protección de los derechos de los ciudadanos y la ingobernabilidad se hacian cada vez más palpables. En el año 2012, justo cuando el Consejo de Estado aceptó el recurso que ordenaba la indemnización a las comunidades, los magistrados de la Corte Constitucional que revisaron la tutela de Epsa y, contrario a lo que se esperaba, le concedieron el amparo a la electrificadora. Solo después de casi quince años de producidos los daños, la Sala Plena de la Corte Constitucional les dió la razón a las comunidades del Anchicayá y a su abogado.

Finalmente, se evidenció una clara contradicción entre los intereses de multinacionales y empresas privadas y los de las poblaciones afrodescendiente e indígena, pues estos últimos son vulnerados frecuentemente y rezagados para la toma de decisiones; por lo tanto, la definición entre gobernados y gobernantes es una relación de tipo asimétrica que facilita el crecimiento de las desigualdades sociales entre los que tienen y los que no. La gobernanza es entonces vertical y la gobernabilidad en esta situación no se ejecutó.

Se determina que el nivel de gobernabilidad es mínimo, pues, a pesar del fallido intento estatal por remediar los impactos veintitrés años después de la construcción de la represa, no hay evidencia del acatamiento de las normas, esta situación se refleja en que las problemáticas son aún latentes en las zonas aledañas a la Salvajina. Además, es inadmisible hablar de gobernabilidad cuando el Estado solo está presente en las comunidades en lo que respecta a seguridad armada. El bienestar social y la garantía de los derechos de la sociedad son relegados, porque los 
actores privados han ocupado el papel del Estado y lo hacen desde una perspectiva económica descarnada que traspasa el tejido cultural de la comunidad.

\section{Conclusiones preliminares}

La CMR designa a "las grandes represas como instrumentos del desarrollo" (CMR, 2002 p.11), pero en su construcción no da cabida a las consecuencias sociales que le son inherentes, pues las asume como transitorias. "El gran instrumento del desarrollo" se ha convertido en un elemento con el que las empresas multinacionales generan un provechoso sustento económico, pues su construcción y producción de servicios representa un gran capital lucrativo. El desarrollo capitalista se ha involucrado directamente en la administración de las represas y en esa medida subordina el bienestar social y la seguridad colectiva de las poblaciones aledañas.

La economía capitalista en el mercado latinoamericano se basa en la mercantilización de las relaciones sociales, ya que acoge un nuevo modelo de sociabilidad fundamentado en el egoísmo y el individualismo de actores privados, que, a su vez, promulga un statu quo en el que la privatización favorece a una esfera de empresarios sobre la sociedad civil, situación reafirmada por el Estado en un marco legal (Lechner, 1996). Tal es el caso de Fenosa que, gracias a las pocas acciones estatales, ha permanecido distante a los reclamos de la comunidad por los impactos de la represa.

El desplazamiento de esferas privilegiadas responde a las interpretaciones neoliberales del individualismo, donde el ámbito público desde su estado comunitario se disipa en el mercado de la acción privada (Lechner, 1996). Hablamos del mercado privado con carácter público, por consiguiente, la conjugación de las esferas que va más allá del concepto interrelacionado de la gobernanza, pues la sociedad civil es relegada completamente frente a un Estado opresor y un mercado fluctuante 
obligado a adaptarse al contexto, en lugar de que sea este el que se adapte a sus requerimientos, con el objetivo de negar las condiciones vitales de la comunidad a cambio del beneficio económico.

La represa Salvajina es un ejemplo latente de una estructura social agonizante, en tanto ha perdido la seguridad que el Estado debería proporcionar; esto se ve representado en las sistemáticas violaciones a derechos humanos y asesinatos a líderes sociales, así como en el desinterés mutuo entre Fenosa y los gobiernos por estudiar y reparar los daños ambientales causados.

Se puede inferir que las comunidades de Suárez, Morales y Buenos Aires, respecto a la problemática de la represa de Salvajina, han entrado en un proceso de razonamiento metonímico propio de la modernidad líquida, donde el comunitarismo ha proporcionado la seguridad para recuperar la acción estatal perdida. Este escenario le ha llevado a agruparse en una relación espacial-causal por las dinámicas de vida (Bauman, 2002). Esto se expresa en pronunciamientos de la sociedad civil, en general las protestas y las luchas de diferentes líderes comunitarios, para abogar por la certeza y la seguridad como habitantes de una zona donde los impactos sociales y ambientales son latentes y olvidados.

Entender que "vivir significa en un lugar, y saber significa, ante todo, saber en qué lugar se está" (Escobar, 2005, p. 57) y que la cultura parte de los cuerpos y los lugares. De lo anterior se comprende que la mayor incidencia a causa de Salvajina se dio en la cultura de los pobladores desplazados por su construcción, pues el lugar y, por consiguiente, el saber que tienen de este ellos, se transformó a partir de nuevos medios de producción; esto generó dinámicas de vida que, hasta el momento, solo giran en torno a la represa.

Las dinámicas de lucha por la represa Salvajina se han culturalizado a causa de los impactos sociales y ambientales de las multinacionales, quienes han hecho de la represa un sustento económico favorable, a tal 
magnitud que reprimir a la sociedad no es más que un método producente y efectivo para el interés privado.

Desde la cultura, se concluye que la construcción de una hidroeléctrica, prioriza el beneficio en términos económicos y de servicios públicos, pero trae consigo afectaciones permanentes a la relación sujeto-tierra, pues engendra daños a una construcción progresiva de prácticas ancestrales que se ven transmutadas por el desplazamiento forzado y otros fenómenos de violencia de hecho y simbólica. Las comunidades anquilosadas viven en la más grande distopía de la modernidad, la muerte lenta y agonizante de su cultura social.

\section{Referencias}

Ararat, Lisifrey; Vargas, Luis Armando; Mina, Eduar; Rojas, Axel; Solarte, Ana María; Vanegas, Gildardo y Vega, Aníbal (2013). La Toma: Historias de territorio, resistencias y autonomía en la cuenca del Alto Cauca En A. Caicedo (ed.). Vida campesina y modelo de desarrollo: configuraciones de despojo /privilegio en el norte del Cauca. Bogotá, Colombia: Universidad de los Andes. Recuperado de http://www.scielo.org.co/pdf/rcan/v53n1/04866525-rcan-53-01-00059.pdf

Bauman, Zygmunt (2000). Trabajo, consumismo y nuevos pobres. Barcelona: Editorial Gedisa.

Bauman, Zygmunt (2002). Modernidad líquida. Buenos Aires: Fondo de Cultura Económica.

Bello, Álvaro (2004). Etnicidad y ciudadanía en América Latina. Santiago de Chile: Cepal.

Betancourt, Santiago (1994). Pugna por el Ovejas. El Liberal.

Canizales, Diego (2013). Factores que inciden en el cambio de una economía licita por una economía ilícita en la zona rural del municipio de Suarez cauca desde 1990-2012. Cali: Universidad del Valle.

Censat (s. f). Proceso de comunidades negras denuncian desalojo del corregimiento La Toma por Anglo Gold Ashanti. Recuperado de http://censat. 
org/es48/noticias/proceso-de-comunidades-negras-denuncian-desalojodel-corregimiento-la-toma-por-anglo-gold-ashanti

Cepal y Patrimonio Natural (2013). Amazonia posible y sostenible. Bogotá: Cepal y Patrimonio Natural.

Colectivo Punto de Fuga (02 de abril de 2012). La salvaje Salvajina, el despojo y el oro en Suárez. Notiagen. Recuperado de https://notiagen.wordpress. com/2012/04/02/la-salvaje-salvajina-el-despojo-y-el-oro-en-suarez/

Comisión Mundial de Represas (CMR) (2000). Represas y desarrollo: un nuevo marco para la toma de decisiones. Recuperado de: https://chiltepines.files. wordpress.com/2012/09/represas_y_desarrollo_sc3adntesis-wcd.pdf

Corporación Autónoma Regional del Cauca (CRC) (2010). Caracterización ambiental plan departamental de aguas y saneamiento básico Departamento del Cauca. Popayán: CRC. Recuperado de http://crc.gov.co/files/ConocimientoAmbiental/AMB_PDA_CAUCA.pdf

Corporación Servicios Profesionales Comunitarios Sembrar (2010). Despojo del territorio en comunidades afrocolombianas. Recuperado de http://jenzera. org/wordpress-content/uploads/2010/01/DESPOJO-DEL-TERRITORIO-ENCOMUNIDADES-AfROCOLOMBIANAS.pdf

Corte Constitucional (2015). Constitución política de Colombia de 1991. Recuperado de http://www.corteconstitucional.gov.co/inicio/Constitucion $\% 20$ politica \%20de \%20Colombia \% 20-\%202015.pdf

CRIC (2013). Implementación de ODM. En C. Duarte (Ed.). Análisis de la posesión territorial y situaciones de tensión interétnica e intercultural en el departamento del Cauca. Cali: Pontificia Universidad Javeriana. Recuperado de https:// www.javerianacali.edu.co/sites/ujc/files/node/field-documents/field_document_file/analisis_posesion_territorial_-_tensiones_interetnicas_e_interculturales_en_el_cauca_1.pdf

Duarte, Carlos (Ed.). (2013). Análisis de la posesión territorial y situaciones de tensión interétnica e intercultural en el departamento del Cauca. Cali: Pontificia Universidad Javeriana. Recuperado de https://www.javerianacali. edu.co/sites/ujc/files/node/field-documents/field_document_file/analisis_posesion_territorial_-_tensiones_interetnicas_e_interculturales_en_el_ cauca_1.pdf 
Escobar, Arturo (2000). El lugar de la naturaleza y la naturaleza del lugar: iglobalización o postdesarrollo? En: Edgardo Lander (Comp.). La colonialidad del saber: eurocentrismo y ciencias sociales. Perspectivas Latinoamericanas. Buenos Aires: CLACSO. Recuperado de http://bibliotecavirtual.CLACSO.org. ar/ar/libros/lander/escobar.rtf

Escobar, Arturo (2005). Más allá del Tercer Mundo. Globalización y diferencia. Bogotá: ICANH.

Escobar, Arturo (2010). Una minga para el postdesarrollo: lugar, medio ambiente y movimientos sociales en las transformaciones globales. Lima: Universidad Nacional Mayor de San Marcos. Recuperado de http://www.unc. edu/ aescobar/text/esp/escobar.2010.UnaMinga.pdf

Escué, Alcibiades (1995). Tierra profanada. Grandes proyectos en territorios indígenas de Colombia. Bogotá: Proyecto ONIC Cecoin Ghk.

Galvis, Catalina (2014). Minería en el norte del Cauca indígena. En B. Göbel y A. Ulloa, (Eds.). Extractivismo minero en Colombia y América Latina. Bogotá: Universidad Nacional de Colombia.

González, Érika (2008). Unión Fenosa. Los impactos de la multinacional eléctrica en Colombia. Bogotá: Observatorio de Multinacionales en América Latina.

González, Fernán y Gama, Claire (Eds.). (2010). Dificultades, retos y posibilidades de la noción de Gobernanza en el caso colombiano. Bogotá: Pontificia Universidad Javeriana.

Guzmán, Álvaro y Rodríguez, Alba (2015). Orden social y conflicto armado: el norte del Cauca 1990-2010. Cali. Universidad del Valle. Recuperado de http://site.ebrary.com.acceso.unicauca.edu.co/lib/biblioucaucasp/reader. action? docID $=11312822$ yppg $=7$

Juanillo, Ismael (1997). Salvajina, oro y pobreza. Popayán: Editorial Antena Comunicaciones.

Lechini, Gladys (Comp.). (2008). La globalización y el consenso de Washington. Buenos Aires: Clacso.

Lechner, Norbert (1996, mayo). ¿Por qué la política ya no es lo que fue? Revista Foro, 29. 
Marín Correa, Alexander (11 de septiembre de 2011). Anchicayá: sin pescado y sin indemnización. El Espectador. Recuperado de http://www.elespectador.com/noticias/judicial/anchicaya-sin-pescado-y-sin-indemnizacionarticulo-298539

Meneses, Ferney (2013). Patía: un proyecto hidroeléctrico que corre en silencio. Censat, Agua Viva. Recuperado de http://censat.org/es/noticias/patia-unproyecto-hidroelectrico-que-corre-en-silencio

Ministerio de Ambiente, Vivienda y Desarrollo Territorial (Minambiente) (2008). "Resolución número 1248 del 11 de junio de 2008". Bogotá.

Movimiento Ríos Vivos (02 de agosto de 2015). Afectados por construcción de represas se están quedando sin territorio Ríos Vivos. Recuperado de https://defensaterritorios.wordpress.com/2015/08/02/afectados-por-construccion-de-represas-se-estan-quedando-sin-territorio-rios-vivos/

Observatorio de Multinacionales en América Latina (Omal) (2012). Presa hidroeléctrica de Salvajina en Colombia: 26 años de despojo. Recuperado de http://omal.info/spip.php?article4737

Osorio, Flor y Betancur, Juan (2013). Participación de grupos étnicos y nuevas agendas del desarrollo en Colombia. En C. Barreira, R. González, J. Zuluaga y J. V. Dos Santos (Coords.) Conflictos sociales, luchas sociales y políticas de seguridad ciudadana. México D. F.: Clacso.

Pegoraro, Juan (2013). El control social como custodio del orden social: selectividad e impunidad de los poderosos. En C. Barreira, R. González, J. Zuluaga y J. V. Dos Santos (Coords.) Conflictos sociales, luchas sociales y politicas de seguridad ciudadana. México D. F.: Clacso.

Roa Avendaño, Tatiana y Duarte, Bibiana (2013). Desarrollo hidroeléctrico, despojo y transformación territorial: El caso de Hidrosogamoso, Santander, Colombia. En A. Arroyo y R. Boelens (Eds.). Aguas robdas. Despojo hídrico y movilización social. Quito: Abya Yala, Justicia Hídrica, Instituto de Estudios Peruanos (IEP). Recuperado de https://totumasymaracas.files.wordpress.com/2013/10/doc_tati-bibi_art-hidrosogamoso_aguas-robadas_2013_rfinal.pdf

Roa Avendaño, Tatiana y Navas, Luisa (Coords.) (2014). Extractivismo, conflictos y resistencias. Bogotá: Censat. 
Ruiz, Pablo (2012). Miles de ciudadanos estadounidenses protestaron contra la Escuela de Asesinos. Rebelión. Recuperado de http://www.rebelion.org/ noticia.php?ID $=159524$

Schorr, Tomas (Ed.). (1984). Las represas y sus efectos sobre la salud. México, D. F.: Centro Panamericano de Ecología Humana y Salud. Recuperado de http://www.cridlac.org/digitalizacion/pdf/spa/doc10207/doc10207.htm

Sulé, Javier (2006). Unión Fenosa en Colombia. Una estrategia socialmente irresponsable. Barcelona: Observatorio de la Deuda en la Globalización, Universitat Politécnica de Catalunya. Recuperado en: https://www.colectivodeabogados.org/IMG/pdf/b36_UnionFenosa_Colombia.pdf

Trejos, Luis (2013). Colombia: una revisión teórica del conflicto armado. Enfoques, 11(18), 55-75.

Vanegas, Gildardo y Rojas, Axel (2012). Poblaciones negras en el norte del Cauca. Bogotá: Departamento de Desarrollo Rural y Regional de la Universidad Javeriana. Observatorio de Territorios Étnicos.

Zapata, Carlos; Nkogo, Eugenio; Proto, Fernando; Esterman, Josef; Brito, Luis; Jordán, María Eugenia et al. (2013). Filosofía mestiza, interculturalidad, ecosofia y liberación. Buenos Aires: Editorial Abierta FAIA. 